\title{
Rabaska
}

Revue d'ethnologie de l'Amérique française

SAIDI, HABIB et SYlVIE SAgnes (dir.). Capitales et patrimoines à l'heure de la globalisation / Capital Cities and Heritage in the Globalization Era. Québec, Les Presses de l'Université Laval, "Patrimoine en mouvement ", 2012, 426 p. ISBN 978-2-7637-9755-7

\section{Cindy Morin}

Volume 11, 2013

URI : https://id.erudit.org/iderudit/1018552ar

DOI : https://doi.org/10.7202/1018552ar

Aller au sommaire du numéro

Éditeur(s)

Société québécoise d'ethnologie

ISSN

1703-7433 (imprimé)

1916-7350 (numérique)

Découvrir la revue

Citer ce compte rendu

Morin, C. (2013). Compte rendu de [SAIDI, HABIB et SylviE SAGnes (dir.).

Capitales et patrimoines à l'heure de la globalisation / Capital Cities and Heritage in the Globalization Era. Québec, Les Presses de l'Université Laval, « Patrimoine en mouvement », 2012, 426 p. ISBN 978-2-7637-9755-7]. Rabaska, 11, 255-257. https://doi.org/10.7202/1018552ar d'utilisation que vous pouvez consulter en ligne. 
Saidi, Habib et Sylvie Sagnes (dir.). Capitales et patrimoines à l'heure de la globalisation / Capital Cities and Heritage in the Globalization Era. Québec, Les Presses de l'Université Laval, «Patrimoine en mouvement », 2012, 426 p. ISBN 978-2-7637-9755-7.

Le colloque international «Capitales et patrimoines au XXI" siècle » s'est tenu à Québec les 5, 6 et 7 novembre 2009. Il était organisé conjointement par l'Institut du patrimoine culturel (IPAC), le Centre interuniversitaire d'études sur les lettres, les arts et les traditions (CÉLAT) et le Laboratoire d'anthropologie et d'histoire de l'institution de la culture (LAHIC). La codirection était assurée par Habib Saidi (IPAC et CÉLAT, Université Laval) et Sylvie Sagnes (CNRS-IIAC, Équipe LAHIC, Paris), qui travaillent tous deux sur le patrimoine de villes capitales.

Les deux coorganisateurs du colloque en signent les actes intitulés Capitales et patrimoines à l'heure de la globalisation et parus en 2012. Dans leur introduction commune, Saidi et Sagnes énoncent la question centrale : il s'agira de « $[\ldots]$ comprendre comment le patrimoine contribue à élever la ville à la dignité de capitale et participe de son identité de "première" et, parallèlement, ce que la capitale "fait" au patrimoine » (p. 16). Voilà ce qui nourrira le lecteur au fil de sa lecture. Suite à la préface de Daniel Fabre, directeur adjoint de l'Institut interdisciplinaire d'anthropologie du contemporain du LAHIC, et à l'introduction, l'ouvrage se divise en trois parties pour se terminer sur la postface du philosophe Olivier Mongin. Les auteurs - des professeurs, chercheurs et directeurs de centres de recherche - enrichissent le collectif de leurs expériences et de leurs horizons disciplinaires variés : ethnologie, anthropologie, histoire, géographie, philosophie, archéologie, etc. Affiliés à des institutions diverses, ils offrent un tour du monde aux lecteurs par leurs études de cas hétéroclites, dont Washington DC, Tel-Aviv, Tunis, Rio de Janeiro. Aucune biographie ne présente plus éloquemment les auteurs. On peut également regretter que l'ouvrage ne comporte pas de bibliographie.

La préface de Daniel Fabre place le cadre des analyses qui suivent en situant l'objet principal de l'ouvrage, à savoir la capitale en général et son centre historique en particulier. À son avis, l'intérêt de travailler sur les capitales réside en deux points : la confrontation directe qui s'impose entre le patrimoine et le politique, l'économie, le spirituel et le religieux ; ainsi que le modèle comparatif qui en résulte, soit une comparaison culturelle, spatiale et temporelle des capitales. Il présente le paradoxe de la capitale qui est réfléchie comme un lieu de confrontation et de contradiction des idéologies temporelles où la récapitulation du passé et de l'histoire nationale entre en conflit avec l'anticipation du futur, c'est-à-dire l'avenir et le destin collectif en passant notamment par la modernisation des services. La simultanéité de ces deux orientations temporelles tendrait à diviser les fonctions et à immo- 
biliser le centre historique, le muséifier et ainsi le vider de ses fonctions et de sa qualité centralisatrice. S'il existe selon Fabre quatre grandes solutions pour pallier ces problèmes de temporalité, « la négociation permanente pour une cohabitation des historicités » (p. 7) l'emporterait par exemple sur la destruction des traces tangibles du passé ou sur le choix d'une temporalité en vertu d'une idéologie dominante.

Saidi et Sagnes, dans leur introduction commune, viennent compléter le portrait de la capitale dans une approche historique et sémantique où la capitale est dite instable, en constante mutation, parfois autoproclamée et toujours potentiellement patrimonialisable. S'ils dessinent minutieusement les contours de la capitale d'hier et d'aujourd'hui, les auteurs s'abstiennent de clarifier le terme de patrimoine rappelant laconiquement que beaucoup a déjà été écrit sur le sujet.

Le cœur de l'ouvrage comporte seize textes divisés en trois parties. La première partie, sous le thème « Capitale nationale, pouvoir et patrimoine ", est composée de cinq textes abordant les rapports entre patrimoine et Étatnation, cette définition déclinante de la capitale, dans le contexte du postnationalisme. La seconde partie, qui regroupe six auteurs, s'intitule « Entre singulier et pluriel, entre particulier et universel : patrimoine mondial et capitale globale ». Elle se penche sur la prédisposition de la capitale à l'universalité et sur la participation du patrimoine à cette affirmation, la reconnaissance par l'UNESCO venant souvent renforcer ce caractère universel vers lequel tendent les capitales par le biais du patrimoine culturel. « Majesté capitulaire, patrimonialité ordinaire et pratiques alternatives du patrimoine » est le thème de la dernière partie qui comporte cinq articles. Les auteurs s'intéressent ici à des patrimoines locaux, impartageables, invisibles ou « incapitularisables » tels que le patrimoine de l'esclavage mis en valeur à Rio de Janeiro dans les musées communautaires.

Qui s'intéresse spécifiquement à l'Amérique française s'attardera davantage sur la deuxième partie du livre, puisque les cas de Québec et de Montréal y sont abordés. D'abord, Guy Mercier aborde les théoriques questions de la mémoire, de l'identité et de la territorialité dans le contexte universel d'une ville déclarée patrimoine de l'humanité par l'UNESCO. Il soulève certains paradoxes, dont celui du lieu de mémoire qui est à la fois un territoire (matériel) habité à aménager et un espace de mémoire (immatériel) pour la société. On peut en effet se demander si un territoire donné peut être vécu et géré par ses habitants, tout en détenant un statut de lieu de mémoire et, qui plus est, de patrimoine universel. Guy Mercier raconte le cas de la ville de Québec où les résidents sont impliqués dans le processus de planification urbaine et où les enjeux relevant du dossier du patrimoine mondial ne sont plus seuls en haut de la liste des préoccupations. 
De son côté, Habib Saidi envisage « le statut "capitulaire" [...] sous l'angle de sa valeur patrimoniale et de son attrait touristique » (p. 235) dans une comparaison Québec/Tunis de l'offre touristique, plus particulièrement des spectacles de rue. Il aborde en premier lieu le changement de paradigme de l'espace urbain et de la capitale à l'ère de la globalisation : l'espace urbain est devenu « agéographique » et réseauté, déhiérarchisant la capitale qui perd ainsi ses statuts de primauté, de suprématie et de centralité. Dans ce contexte, la capitale ne se résume plus aux rôles politique et administratif, et des villes non-capitulaires se trouvent capitalisées, augmentant ainsi la concurrence entre les villes de tout acabit. Cependant, ce que la mondialisation a fait perdre à la capitale est récupéré via le tourisme et le patrimoine, les villes recourant à ce dernier pour se différencier dans leur offre touristique. Le patrimoine est ainsi utilisé comme axe de convergence et comme affirmation de la localité dans un contexte globalisant. En résumé, « la globalisation a déchu la capitale de son statut de centre de pouvoir et de ville du trône, le tourisme [par l'entremise du patrimoine] l'a dotée du statut de destination la plus représentative, par rapport à d'autres villes, de l'identité nationale et de ses emblèmes. » (p. 255).

Finalement, un article de Sylvie Sagnes sur le cas de Montréal complète le trio de textes se rapportant de manière spécifique à l'Amérique française. Comme mentionné en fin de texte, la muséographie de la montréalité constitue le sujet de cette étude qui écarte l'objet patrimonial et la question centrale posée en introduction. L'auteur s'affaire d'abord sur l'histoire ancienne de la défaite de Montréal pour l'obtention d'un titre officiel de capitale politique puis sur ses nombreux statuts actuels de capitale de toute sorte (de la francophonie en Amérique, des arts du cirque, du multimédia, etc.) dans le contexte globalisant déjà abordé. Après une longue mise en contexte historique, statistique et socioculturelle de la ville et de sa montréalité, l'auteur statue sur la « capitularité d'un autre genre » (p. 222) de Montréal. La question du rôle du patrimoine dans l'avenir transculturel de Montréal est ensuite explorée par le biais de l'étude de l'exposition Les amours de Montréal, exposition abordant les diverses identités montréalaises d'aujourd'hui.

Pour conclure, cet ouvrage discute de la capitale dans le contexte de la globalisation, thèmes abondamment analysés par plusieurs auteurs. Quant à la vaste notion de patrimoine, elle revêt plusieurs aspects au gré des études passant de l'héritage architectural et des monuments de la ville aux questions identitaires ou de mémoire.

Cindy Morin

Consultante en patrimoine, Montréal 